

Universidad Santo Tomas

Facultad Administración de Empresas

INFORME FINAL PERIPLO INTERNACIONAL UVERSIDAD DE SALAMANCA ESPAÑA

\title{
APROXIMACIÓN TEÓRICA SOBRE EL IMPACTO DEL LIDERAZGO EN LA ROTACIÓN DEL PERSONAL EN UN SUPERMERCADO DE BOGOTÁ
}

Autores:

Karen Garzón, Carlos González, Jhon Guarnizo.

Tutores:

Profesores. Jairo Contreras, Luis Moreno.

Bogotá, 22 Enero de 2020 
Tabla de contenido

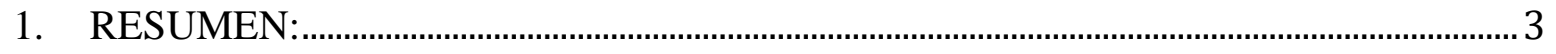

2. PALABRAS CLAVES:

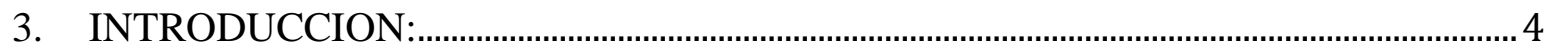

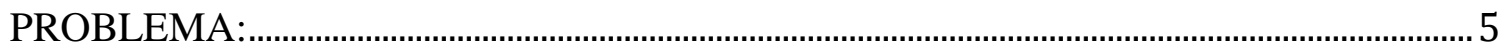

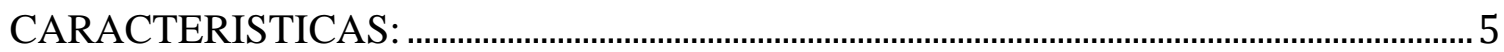

PREGUNTA PROBLEMA: ........................................................................................................ 5

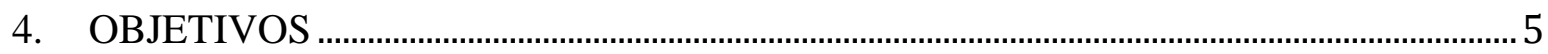

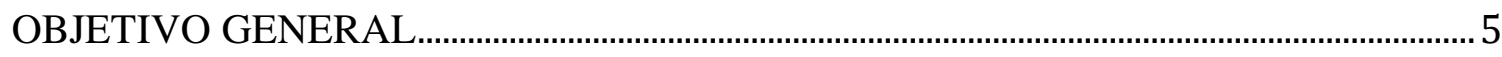

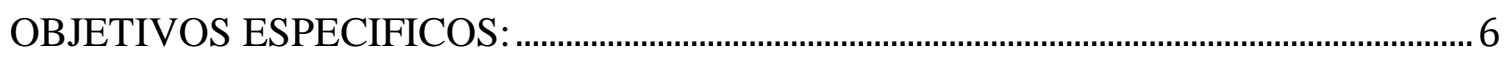

5. Contextualización..................................................................................................................... 6

6. REFERENTES TEORICOS O CONCEPTUALES: .........................................................

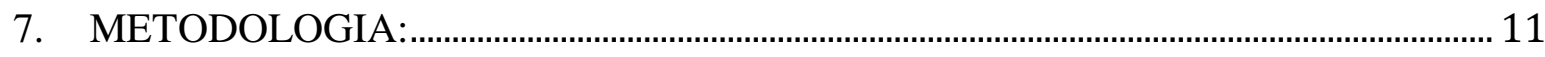

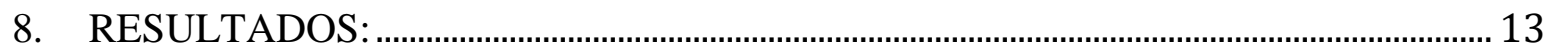

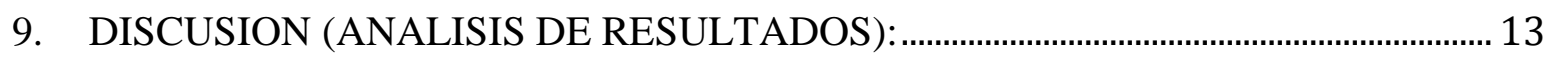

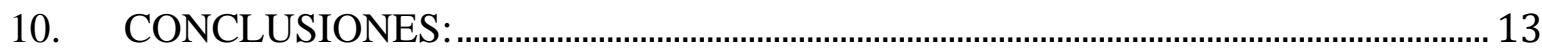





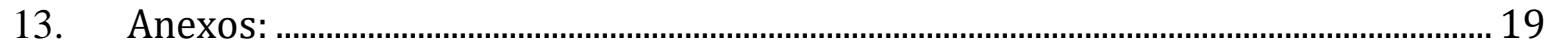

\section{LISTA DE TABLAS}

I. indicadores económicos

II. Distribución trabajadores Grupo Comercial Siglo XXI

III. Índice de rotación Grupo Comercial Siglo XXI

IV. España vs Colombia (Supermercado)

\section{LISTA DE FIGURAS}

I. Modelo de coaching Co-Activo para el liderazgo en la empresa 


\section{RESUMEN:}

Este informe busca ser un insumo para una posible mejora dentro de una empresa de supermercados ubicada en la ciudad de Bogotá, donde se aproxima teóricamente la importancia del liderazgo en las empresas familiares.

Su principal actividad es realizada con supermercados cubriendo gran parte de la canasta familiar. Su gran falencia es la poca preparación de los lideres, ya que son personas que no cuentan con la oportunidad de estudiar, sino se van formado en el tiempo con las actividades que realizan a diario en el trabajo.

Esto lleva a querer estudiar un poco mas a fondo este termino ${ }^{1}$, y poder brindar por medio de la actividad académica ${ }^{2}$ y una aproximación teórica, de todo lo nombrado anteriormente.

\section{PALABRAS CLAVES:}

Liderazgo, comunicación, empresa, equipo

\section{ABSTRACT:}

This report will be an input for a possible improvement inside a supermarkets company located in Bogotá, where the importance of leadership is theoretically approached on family businesses.

Its main activity is carried out with supermarkets covering a large part of the family basket. Its great flaw is the poor preparation of the leaders, since they are people who do not have the opportunity to study, but are trained in time with the activities they perform daily at work. This leads to wanting to study this term a little more thoroughly, and to be able to provide through academic activity and a theoretical approach, of everything previously mentioned.

KEYWORDS:

Leadership, communication, company, team

${ }^{1}$ Liderazgo Transformacional.

2 Periplo Internacional Universidad de Salamanca "Liderazgo y talentos en las empresas del siglo XXI “ Diciembre - 2019. 


\section{INTRODUCCION:}

El desempeño de las organizaciones se ve ampliamente influenciado por el rendimiento, las habilidades y la eficiencia de las personas que trabajan allí. En la actualidad se conoce que este factor puede ser un diferenciador y una ventaja competitiva para la empresa dentro del mercado y para todos sus stakeholders. Por esto, es de suma importancia abordar a las personas como potencializadores de las organizaciones por lo cual se considera que la habilidad del liderazgo es fundamental para que estas ventajas que las personas pueden aportar, sean aprovechadas por las empresas.

Según lo anterior, y en el contexto del desarrollo del liderazgo dentro de las organizaciones, se busca determinar que estrategias podrían ser convenientes para que los nuevos emprendimientos puedan gestionar el talento de su organización y puedan tanto obtener ventajas competitivas como retener a sus trabajadores y así hacer más eficiente su operación. Aterrizando el informe al plano real, los conceptos mencionados serán relacionados según el caso de una empresa que cuenta con cuatro Supermercados ubicados en el norte de la ciudad de Bogotá, los cuales cubren desde la calle ciento sesenta y tres (163) hasta la calle ciento ochenta y nueve (189), la cual presenta actualmente una problemática de falta de liderazgo y alta rotación de las personas.

La empresa fue conformada de forma empírica; actualmente esta se ha consolidado como un grupo económico activo. Cuenta con personal administrativo y operativo. Desde el área de talento humano en dos mil diecinueve (2019) surgió la necesidad de formar a todos los trabajadores en pro de tratar los siguientes problemas evidenciados dentro de la organización. 


\section{PROBLEMA:}

La empresa ${ }^{3}$ carece de personal con liderazgo para poder manejar y hacerse responsable de un punto de venta, teniendo así problemas para cumplir los objetivos que la alta gerencia propone.

\section{CARACTERISTICAS:}

1. Personal con poca preparación académica, teniendo que adquirir la formación en su lugar de trabajo, generando retrasos en los procesos.

2. Pocos incentivos de parte de la empresa para que los trabajadores se comprometan.

3. Falta de direccionamiento, para generar un aprendizaje adecuado con lo que se quiere.

Por lo tanto, a través de este informe y mediante una aproximación teórica de las tendencias en cuanto a la mejora del liderazgo organizacional, se espera sugerir a esta empresa de supermercados herramientas y actividades acordes a su contexto que podrían a futuro ser implementadas en la organización con el fin de solventar los inconvenientes que presentan actualmente con relación al talento humano de la compañía.

\section{PREGUNTA PROBLEMA:}

¿Cuáles son las herramientas que pueden fortalecer el liderazgo y disminuir la rotación de personas en las empresas familiares?

\section{OBJETIVOS}

\section{OBJETIVO GENERAL}

Establecer cuales herramientas pueden ser de útiles para mejorar las habilidades de liderazgo que permitan disminuir los índices de rotación de la empresa de supermercados

${ }^{3}$ Grupo Comercial Siglo XXI S.A.S 


\section{OBJETIVOS ESPECIFICOS:}

1. Identificar las características que llevan al liderazgo transformacional hacer el preferido en el siglo XXI.

2. Definir al coaching como herramienta de mejora del liderazgo transformacional dentro de las organizaciones

3. Advertir de la importancia de la retención del talento en las empresas modernas y como esto mejora el funcionamiento de las organizaciones

\section{Contextualización}

La unión europea es una organización que se da inicio después de la segunda guerra mundial en el año 1958 con la cooperación de seis países, el objetivo de esta unión era impulsar la economía incentivando el comercio y disminuir el conflicto, en la actualidad cuenta con veintiocho miembros la unión europea. España se integra a la UE en 1985 "firma del Tratado de Adhesión en Madrid y la integración efectiva en la Comunidad Económica el 1 de enero de $1986 "$

España, aunque en sus indicadores económicos no demuestra un crecimiento significativo, los habitantes tanto nacionales como extranjeros valoran otras variables como la seguridad, el clima y la calidad de vida posicionándolo en el cuarto mejor país para vivir, pero no para prosperar, el aspecto económico no resulta especialmente atractivo ya que la renta media de 36.000 euros (41.000 dólares), se sitúa en un escalafón relativamente bajo frente a los 67.000 euros (76.000 dólares) de la media mundial.

Tabla 1 indicadores económicos

\begin{tabular}{|l|l|}
\hline$\underline{\text { IPC }}$ & $\begin{array}{l}\text { La tasa de variación anual del IPC en } \\
\text { España en octubre de 2019 ha sido del 0,1\%, } \\
\text { con lo que se repite el dato del mes anterior. } \\
\text { La variación mensual del IPC (Índice de } \\
\text { Precios al Consumo) ha sido del 1\%, de } \\
\text { forma que la inflación acumulada en } 2019 \\
\text { es del 0,7\%. }\end{array}$ \\
\hline$\underline{\text { PIB }}$ & $\begin{array}{l}\text { PIB interanual es del 2\% con un aumento } \\
\text { trimestral del 0.4\% y España tiene como } \\
\text { peso del 11\% del PIB debido al turismo }\end{array}$ \\
\begin{tabular}{l|l} 
España tiene un PIB Per cápita trimestral de \\
$6.629 €$ euros, 185 euros mayor que el del \\
mismo trimestre del año anterior, que fue de \\
6.444 euros.
\end{tabular} \\
\hline$\underline{\text { Deuda externa }}$ & $\begin{array}{l}\text { La deuda externa bruta de España se situó } \\
\text { en 2,058 billones de euros durante el primer } \\
\text { trimestre, con lo que escala hasta el 169\% } \\
\text { del PIB, según los datos del Banco de }\end{array}$
\end{tabular}


Con el propósito de establecer un referente de mejora en términos de liderazgo para la empresa objeto de estudio, se realiza a continuación un paralelo con la cadena de supermercados española "Mercadona"

Tabla 2 Paralelo supermercados

\begin{tabular}{|c|c|}
\hline Colombia & España \\
\hline $\begin{array}{c}\text { Descripción } \\
\text { Supermercado objeto de estudio }\end{array}$ & $\begin{array}{l}\text { Descripción } \\
\text { Mercadona }\end{array}$ \\
\hline $\begin{array}{l}\text { - Supermercados de barrio, con } \\
\text { presencia únicamente en el Norte } \\
\text { de la cuidad de Bogotá, cuenta } \\
\text { con } 4 \text { sedes ubicadas } \\
\text { estratégicamente desde la calle } \\
163 \text { hasta la calle } 189 \text { formadas } \\
\text { en el año } 2003 \text {. } \\
\text { - Actualmente es manejada por su } \\
\text { fundador y } 2 \text { de sus hijas } \\
\text { ubicadas en el área de compras y } \\
\text { de recursos humanos } \\
\text { respectivamente. } \\
\text { Desde el área de recursos } \\
\text { humanos se ha enfocado desde } \\
2019 \text { en buscar diferentes } \\
\text { beneficios para los trabajadores, } \\
\text { ya que se ha observado que } \\
\text { existen varias falencias que no } \\
\text { deja avanzar en el cumplimiento } \\
\text { de los objetivos, cabe aclarar que } \\
\text { las áreas de compras y mercadeo } \\
\text { manejan actividades que } \\
\text { sobresalen e impulsan a su } \\
\text { sostenimiento y a enseñarles el } \\
\text { valor más importante que son los } \\
\text { clientes. }\end{array}$ & $\begin{array}{l}\text { - Creada en } 1977 \text { por una pareja } \\
\text { como carnicerías siendo un } \\
\text { negocio familiar y se transforman } \\
\text { los ultramarinos. } \\
\text { - Se crece la sociedad y entre } \\
\text { hermanos le compran a los } \\
\text { fundadores } 8 \text { tiendas y empiezan } \\
\text { su trabajo como independiente, a } \\
\text { través del tiempo van creciendo } \\
\text { las tiendas ubicadas en toda } \\
\text { España con } 10.000 \text { trabajadores y } \\
150 \text { tiendas. Van implementando } \\
\text { estrategias de mercadeo y su } \\
\text { principal actividad precios bajos } \\
\text { con marcas propias. } \\
\text { En 2010 crean un modelo de } \\
\text { gestión de recursos humanos } \\
\text { basado en el liderazgo y la cultura } \\
\text { del esfuerzo para ser una empresa } \\
\text { de alto rendimiento y } \\
\text { productividad. } \\
\text { Los trabajadores de Mercadona } \\
\text { su principal función es satisfacer } \\
\text { al "JEFE" así llaman al cliente, } \\
\text { su principal política es tener un } \\
\text { trabajo estable y de calidad, }\end{array}$ \\
\hline
\end{tabular}




\begin{tabular}{|c|c|}
\hline $\begin{array}{l}\text { - La persona encargada de recursos } \\
\text { humanos se ha preocupado por } \\
\text { tener estabilidad en los cargos, } \\
\text { implementando actividades de } \\
\text { fidelización a la empresa, pero } \\
\text { surge la necesidad de formar } \\
\text { personas para el futuro y para que } \\
\text { esta organización se mantenga en } \\
\text { el tiempo con los cambios } \\
\text { presentados de manera muy } \\
\text { rápido en la actualidad, Cada } \\
\text { punto de venta cuenta con un } \\
\text { líder de almacén el cual es el } \\
\text { responsable de su grupo de } \\
\text { trabajo y de controlar las } \\
\text { actividades que realiza cada uno } \\
\text { que lo conforma, es por eso que } \\
\text { se quiere brindar unas actividades } \\
\text { para practicar con los } \\
\text { trabajadores aplicadas con } \\
\text { nuevas tendencias y asi ir } \\
\text { mejorando el rendimiento. }\end{array}$ & $\begin{array}{l}\text { ofreciendo formación, } \\
\text { promoción interna y la equidad, } \\
\text { reciben incrementos salariales } \\
\text { tras años de antigüedad, cuando } \\
\text { hablan de equidad no les importa } \\
\text { el sexo sino a misma } \\
\text { responsabilidad, mismo sueldo. } \\
\text { - Mercadona comparte la } \\
\text { información y objetivos de la } \\
\text { empresa con sus trabajadores y } \\
\text { para desarrollar el talento de las } \\
\text { personas fomentan el dialogo y la } \\
\text { participación, esto con el fin de } \\
\text { aportar nuevas ideas y } \\
\text { aprovechar la creatividad de cada } \\
\text { uno de los trabajadores, su } \\
\text { principal lema es "para poder } \\
\text { satisfacer a sus clientes } \\
\text { Mercadona también busca } \\
\text { satisfacer a sus trabajadores" con } \\
\text { lo nombrado anteriormente. }\end{array}$ \\
\hline
\end{tabular}

Fuente elaboración propia

\section{REFERENTES TEORICOS O CONCEPTUALES:}

Con el propósito de desarrollar un enfoque hacia el liderazgo para determinar la importancia de este en el funcionamiento de una organización, se destaca una definición de que es el Liderazgo.

"es la influencia interpersonal ejercida en una situación, dirigida a través del proceso de comunicación humana a la consecución de uno o diversos objetivos específicos" Idalberto Chiavenato(2001,p.60)

El liderazgo permite identificar, facultades para poder guiar a un grupo de personas con intereses similares y así cumplir objetivos específicos. En la actualidad se puede identificar varios tipos de liderazgo uno de ellos es el transformacional o motivador, su principal facultad es darle la importancia a las personas en la organización y así se pueda desarrollar un gran potencial. 
El Liderazgo Transformacional es "ejercido por aquellos individuos con una fuerte visión y personalidad, gracias a la cual son capaces de cambiar las expectativas, percepciones y motivaciones de sus seguidores, así como liderar el cambio dentro de una organización" James MacGregor.

Este concepto llevo a desarrollar ciertas características que lleva hacer un gran líder.

1. Estimulación Intelectual: este permite que los trabajadores piensen en el momento de un problema a buscar la mejor solución, involucrándolos para dar sus opiniones y así desarrollarse.

2. Consideración Individualizada: se evalúa a cada persona de manera individual, y se utiliza la inteligencia emocional para que estos se puedan relacionar en equipo.

3. Inspiración y Motivación: el líder es el guía, y los participantes sienten inspiración y motivación para seguir a su guía.

4. Influencia Idealizada: las personas del grupo ven al líder como un ejemplo a seguir, permitiendo que estos se motiven mas a conocer sobre sus intereses, y aportar lo mejor de cada uno.

Se puede identificar que en este tipo de liderazgo se interesan mas por las personas y se desea que estas progresen y expresen sus ideas, la comunicación es vital para llevar a cabo las actividades, ya que este llega a crear un vinculo emocional, para compartir la misma visión, colaborándose entre si y la formación va siendo en la misma dirección, permitiendo que la motivación por lo que hacen, permiten tener un ambiente laboral agradable.

Hay que resaltar que en la actualidad los millenials, buscan que lo que ellos hagan este relacionado con sus intereses, ellos desean tener guías en su carrera que los retroalimenten con respecto a sus logros y sus mejoras.

Esto fue lo que se desarrollo en el seminario en España, identificando características principales de un líder, con trabajo en equipo y como es la actitud tanto personal, como grupal para desarrollar un problema, analizando que el bien común es vital para que se desarrollen los objetivos, y escuchar a cada una de las personas ofrece beneficios, e interés dentro del grupo y poder cumplir los objetivos.

En este contexto se puede sugerir crear el valor de las personas en las empresas familiares, para así poder cumplir objetivos claros, y lograr trabajar en equipo, todo esto conociendo a cada persona, sus necesidades y las metas que desean cumplir.

Según ASESCO el coaching es la herramienta la cual permite el desarrollo individual, fortalecimiento de sus habilidades y la proyección de las metas futuras, esto permite a las 
personas tener una idea clara de lo que desea en un futuro y el proceso que debe realizar para el cumplimiento de estos objetivos tanto profesionales como laborales.

El coaching empresarial tiene como finalidad "incrementar la competitividad empresarial que estimule una cultura de innovación en cada uno de sus colaboradores, lo cual ayudará a obtener los resultados propuestos de acuerdo a sus lineamientos estratégicos" (Batanero, 2010: p. 5). Los trabajadores dentro de una organización se tienen en cuenta como un conjunto o sistema y no como seres por separado.

La clasificación del coaching es según el ámbito de aplicación, esta herramienta tiene varios tipos los cuales se dividen en según el número de personas, contenido y método. Para efectos del estudio se tendrá en cuenta el coaching dirigido de manera individual o en grupo ${ }^{4}$, con características diferenciales, lo cual las empresas valoran dentro de la organización. En ese sentido un coaching ideal para ello es el coaching CO-ACTIVE ${ }^{5}$ para la formación, desarrollo laboral y profesional.

Figura 1: Modelo de coaching Co-Activo para el liderazgo en la empresa

\section{Modelo de Coaching Co-Activo para el Liderazgo en la empresa.}

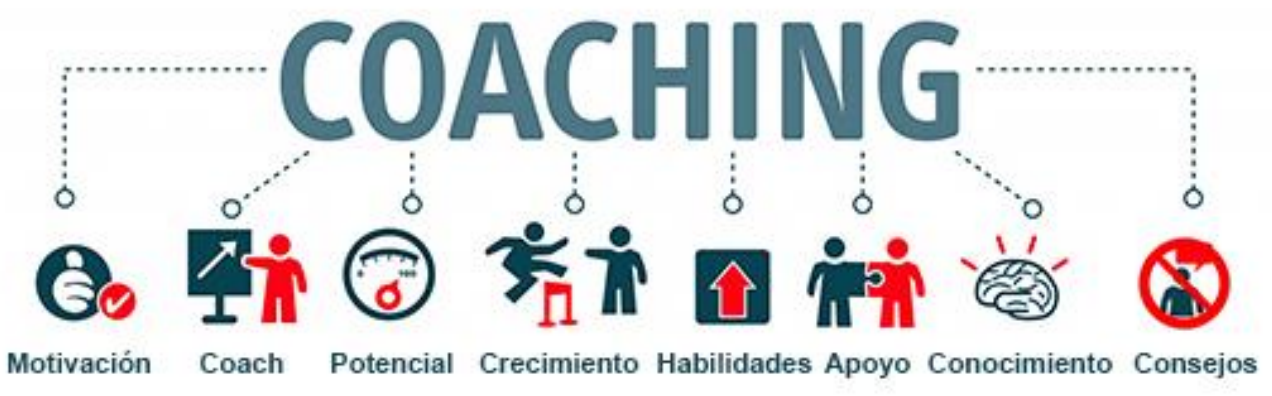

Fuente : Retrieved 22 January 2020, from https://mx.coactive.com/liderazgo-en-la-empresa/

\footnotetext{
${ }^{4}$ En este informe las características del grupo de personas o individuos tendrán en cuenta a los milleniams.

5 "El modelo de coaching co-activo es un enfoque probado que se basa en muchos años de experiencia trabajando con clientes y coaches en todo el mundo. Este libro describe con detalle el modelo, define las habilidades y las técnicas del coaching co-activo, y presenta ejemplos de conversaciones, así como ejercicios prácticos que te permitirán comprenderlo". (Whitworth, Sandahl, Kimsey-House, Kimsey-House \& Martínez Solimán, n.d.)
} 
Este modelo se basa en la relación entre el coach y coaches el cual permite una mejora de las habilidades de los individuos (en este caso de los millennials). El método de este enfoque se caracteriza por realizar preguntas muy efectivas, tiene una escucha activa, impulsa las habilidades y capacita en los temas más relevantes para los trabajadores.

Según Henry Kimsey-House Los cuatro pilares del coaching co-activo son estos:

1. Las personas, por naturaleza, son creativas, completas y están llenas de recursos.

2. El coaching ha de centrarse en la totalidad de la persona.

3. Escuchar atentamente a todos los niveles.

4. Suscitar la transformación.

Teniendo en cuenta que "las empresas internacionales se han convencido que la retención estratégica de los empleados, requiere de su atención prioritaria, así como lo es su mercadotecnia y sus ventas.” (Barragán, 2009), es fundamental resaltar la importancia por retener y potenciar a las personas que se encuentran dentro de la organización, ya que se considera este un factor esencial para el crecimiento tanto de la organización como para las personas en si en su ámbito profesional.

"Las organizaciones no pueden darse el lujo de mantener empelados insatisfechos, ya que la verdadera naturaleza de la visión y misión se lleva a cabo mediante empleados eficientes, productivos y orientados al cliente, y, por lo contrario, la insatisfacción de los empleados impacta directamente en los resultados operativos” (Barragán, 2009, citado por, Ríos y Uribe, E. 2017. P. 30)

Por tanto, las condiciones de satisfacción de los talentos de la organización se deben tener presente estos aspectos, en tanto que esta se vera reflejada en proporción directa con todas las labores que desempeñen los colaboradores en su día a día.

\section{METODOLOGIA:}

Se realizo un estudio explicativo, donde se identifico problema de los líderes de tienda en una empresa, por falta de conocimiento del cargo, se analiza la situación por medio de un factor importante para el manejo de las organizaciones y que hoy por hoy lleva al cumplimiento de los objetivos.

Tomando como punto de referencia una empresa de supermercados que lleva aproximadamente 16 años en el mercado y cuenta con 56 trabajadores distribuidos de la siguiente manera: 
Tabla 2: Distribución trabajadores según cargo

\begin{tabular}{|l|l|}
\hline \multicolumn{1}{|c|}{ Area: } & \multicolumn{1}{c|}{$\mathbf{N}^{\mathbf{0}}$ Trabajadores: } \\
\hline Administrativa & 13 \\
\hline Operativa & 37 \\
\hline Logística & 6 \\
\hline
\end{tabular}

Fuente: elaboración propia

La empresa objeto de estudio, se encuentra ubicada en Colombia en la ciudad de Bogotá, esta trabaja con personas de estratos 1,2 y 3 , en gran mayoría personas con solo el bachillerato, evidenciando un problema en el momento de tomar un cargo alto, ya que su principal requisito para ser encargado de tienda es que lleve de 10 a 15 años en esta, tomando bastante tiempo en su preparación, y por su alto índice de rotación:

Tabla 3 Índice de rotación de personal

\begin{tabular}{|l|l|}
\hline \multicolumn{1}{|c|}{$\mathbf{N}^{\mathbf{0}}$ Trabajadores } & \multicolumn{1}{c|}{ cantidad } \\
\hline Total Planta & 56 \\
\hline Retiros mes & 5 \\
\hline Ingresos mes & 5 \\
\hline
\end{tabular}

Fuente: elaboración propia

En el último cuadro, se puede observar que la rotación aproximada de personal al mes corresponde al $8 \%$ del total de la nómina, este porcentaje es muy alto teniendo en cuenta la cantidad de empleados que trabajan en la empresa. Esto se puede deber a factores como: la baja motivación, la obligación al ir a trabajar por necesidad, los incentivos para su retención, baja capacitación en los cargos.

Dadas estas circunstancias, para fortalecer el liderazgo se propone el coaching con la finalidad de disminuir la rotación de los millennials en la organización.

Inicialmente, se deben realizar procesos que permitan crear una relación fuerte entre el coach y los trabajadores, para ello el primer paso consistirá en la escucha activa puesto que las personas son creativas con nuevas ideas. En el segundo tendrá como propósito profundizarescuchar, para lo cual el coach tendrá que centrarse en la pregunta “¿Cuál es el problema que hay que resolver?", con el propósito de evidenciar la forma como la persona refleja las metas y objetivos que desea cumplir. Finalmente el tercer paso, tiene por finalidad generar curiosidad con una serie de preguntas para generar buen coaching. 


\section{RESULTADOS:}

Para fortalecer el liderazgo en la empresa colombina, se requiere implementar la herramienta del coaching CO-ACTIVO que promueva un Feedback continuo entre los trabajadores y los líderes de la empresa, así mismo, tendrá que crear una política orientada a desarrollar programas en sus colaboradores con capacitaciones para su desarrollo personal y profesional para ampliar sus perspectivas profesionales y personales alineadas a los objetivos estratégico de la empresa. Este tipo de prácticas se reflejan en un personal con sentido de pertenencia por la organización y con esto "la disminución en los indicadores de la rotación del personal".

\section{DISCUSION (ANALISIS DE RESULTADOS):}

El concepto de liderazgo y sus efectos en una disminución en la rotación de personal de la empresa estudiada, se puede dar siempre y cuando se emplee un coaching Coactivo. Estos aspectos deben señalar los programas de mejora de personal en la empresa estudiada que se mantenga en el tiempo. Con esto se esperaría contar con trabajadores creativos e innovadores, cien por ciento motivados y en constante crecimiento personal y profesional

En caso de no tener en cuenta estos aspectos en términos de costos beneficio, la empresa podría tener sobrecosto de nómina hasta de un $25 \%$ del sueldo asignado a cada joven, por concepto del proceso de reclutamiento, contratación y capacitación, una pérdida del $30 \%$ de la productividad total, por la desconfianza, miedo, incertidumbre que causa la salida repentina de los millennials y un 50\% de lo invertido por las organizaciones en sueldos, prestaciones, proyectos y falta de resultados que provoca la rotación y la falta de comunicación laboral. (Enríquez Duarte, 2018)

Estos porcentajes alertan a las empresas a la hora de seleccionar al personal, debido a la incertidumbre que generan, en especial las nuevas generaciones de trabajadores

\section{CONCLUSIONES:}

- El liderazgo es una habilidad que permite desarrollar las actividades de largo y corto plazo en las organizaciones, ya que su principal actividad es brindar orientación a los grupos de interés y así poderlos sintonizar con el cumplimiento de objetivos.

- Al pasar el tiempo las tendencias van avanzando y tener un liderazgo adecuado en cada momento, permite mantenerse en el mercado, por mucho mas tiempo, y genera 
estabilidad en los trabajadores, al sentirse miembros aportan el 100\% de su esfuerzo, logrando tener beneficios mutuos.

- El coaching como herramienta nos da una perspectiva mas amplia frente a la importancia del liderazgo en las organizaciones, pero no solo un líder de toma de decisiones o de control de los procesos. Sino un apoyo con los conocimientos adecuados para realizar un coaching empresarial efectivo, el cual centra en el desarrollo, la motivación, las habilidades entre otras que generan un valor significativo para las personas como para la organización

- Las empresas se centra mucho en la productividad por lo que realizan inversiones en la infraestructura de la organización pero no en su gestión interna con sus trabajadores, debido a que este tipo de inversiones lo proyectan como un gasto que no genera valor, pero en realidad es igual o un grado mas importante para su funcionamiento debido a que el recurso humano es el motor de las empresas, si este no tiene procesos de crecimiento las organizaciones tienden a quedar atrás frente a sus competidores.

- La empresa debe dar mayor relevancia a la retención de su personal ya que la rotación que presenta actualmente genera esfuerzos adicionales en materia de tiempo, implementación repetitiva de procesos, capacitaciones además de los costes que el proceso de selección acarrea.

- Ante el índice de rotación de los empleados de la empresa, se propone que la herramienta del coaching enfocada al liderazgo sea una pieza importante dentro de los procesos de mejora del talento con el fin de que estos líderes potenciales se apropien de sus puestos de trabajo y presenten mayor compromiso y crecimiento para la empresa.

\section{BIBLIOGRAFIA}

Barragán, J., J. Castillo, P. Villalpando \& P. Guerra,UANL, San Nicolás, N.L (2009) Recuperado en México . Recuperado en: http://eprints.uanl.mx/12509/1/A3.pdf

Benavides, S. (2013). El coaching en el ámbito empresarial. Retrieved 22 January 2020, from https://dspace.uib.es/xmlui/bitstream/handle/11201/3599/Benavides_Hena res_Sandra.pdf?sequence $=1$

Casado, B (2010), Manual Coaching, España Madrid, Editorial CEP, S.L.

Cañeque,M, (2017/01/01), El Nuevo Liderazgo, Argentina Buenos Aires, Ediciones Granica

Chiavenato,I (2001), Administración Proceso Administrativo, Colombia, Mc Graw Hill 
Gutiérrez, O, (2015), Revista Política y estratégica No126, Pg. 4, 7, 13.

Hidalgo, A. (2019), Innovación y Mercados Financieros, España Salamanca, Universidad de Salamanca.

Joseph A. Litterer, JL, (1979), Análisis de las Organizaciones, México, Editorial Limusa.

Lozano, B. (2019), Gobierno de la Empresa, España Salamanca, Universidad de Salamanca.

Mendoza, M. (2018). Millennials provocan fuga de 30\% en productividad. Retrieved 22 January 2020. Recueperado en https://www.publimetro.com.mx/mx/noticias/2018/08/14/millennials-provocanfuga-30-productividad.html

McGregor, Douglas (1960), The Human Side of Enterprise New York, McGrawHill

Mercadona (2020). Trabajadoras y trabajadores de Mercadona. España info Mercadona.es Recuperado en: https://info.mercadona.es/es/conocenos/empleo/trabajadoras-ytrabajadores-de-mercadona

Muñoz, P (2019), La Innovación y el Marketing en el Desarrollo de negocios en el mundo digital, España Salamanca, Universidad de Salamanca.

Ogliastri, E, McMillen, C., y Otros en "Cultura y liderazgo organizacional en 10 países de América Latina. El estudio Globe." Academia. Revista Latinoamericana de Administración, no. 22 (1999):29-57. Redalyc, Recuperado en https://www.redalyc.org/articulo.oa?id=71602203

Pindado, J. (2019), Dirección Estratégica y Modelos de Negocios Socialmente Responsables, España Salamanca, Universidad de Salamanca.

Rodríguez, F (2019), Industria Sostenible, España Salamanca, Universidad de Salamanca.

Thomas, B. (2009), Administración: Liderazgo y Colaboración en un mundo competitivo, México D,F., Mc Graw Hill.

Whitworth, L., Sandahl, P., Kimsey-House, H., Kimsey-House, K., \& Martínez Solimán, M.(2014) Coaching co-activo (3rd ed.).

Zapata, S. (2012). ¿Qué Es El Coaching? Sus Orígenes, Definición, Distintas Metodologías Y Principios Básicos De Actuación De Un Coach. Retrieved 22 January 2020.Recuperado en: https://www.3ciencias.com/wpcontent/uploads/2012/06/3.Que-es-Coaching.pdf 


\section{Diario de Campo:}

\section{- Dirección estratégica y modelos de negocio socialmente responsables:}

La idea principal del expositor frente a esta temática se baso en los modelos de negocios, la herramienta principal para proyectar el modelo con la propuesta de valor como su eje para una diferenciación en el mercado, ejemplos como la empresa familiar Mercadona que por su propuesta hace frente a grandes plataformas como carrefourt.

Se da inicio con la definición de que es una estrategia del cual tomaremos que es relacionarse ventajosamente con el entorno y a diferencia de direccionamiento estratégico tiene ciertos elementos adicionales que lo conforman los objetivos estratégicos, los objetivos operativos, acciones vinculadas e indicadores claves, el cual contiene una definición mas amplia a la que anteriormente se tenia de direccionamiento estratégico.

La herramienta administrativa que se tomo para reflejar la creación de una idea de negocio fue el modelo canvas, para dar una propuesta de valor al modelo de negocio, la empresa que se tomo de ejemplo fue la universidad y en ciertos ítems la empresa familiar Mercadona, el docente expositor frente a su experticia propuso una manera diferente en como realizar el modelo de su inicio a fin pasando por sus 9 etapas.

Su 1 etapa a realizar es al segmento de mercado, enfocar quienes son nuestros posibles clientes de este punto nos dará claridad sobre la población a la cual nos vamos a dirigir, la ubicación y las preferencia de la población a la cual queremos llegar, la 2 etapa la propuesta de valor es esa diferenciación en nuestra idea de negocio que vamos a ofrecer a nuestros posibles clientes, 3 etapa CANALES la cual hace referencia a los medios por los cuales se llegaran a los potenciales clientes, 4 etapa relaciones con los clientes este depende mucho del tipo de producto y las necesidades del cliente, lo podemos hacer automatizado o personalizado, 5 etapa fuentes de ingresos respondiendo a la pregunta, ¿Dónde saldrá los cobros?, de los productos o servicios ofrecidos, la diferencia entre ingresos y cobros es que el primero es cuando se realiza la facturación, el cobro es cuando se realiza el pago de esta. la 6 y 7 etapa están muy relacionadas que son las actividades claves y los recursos claves son parte fundamental de la empresa debido a que son los procesos, elementos o materiales fundamentales para el funcionamiento del negocio, 8 etapa asociaciones claves las alianzas 
o acuerdos con nuestros skateholders y por ultimo en el modelo canvas tenemos la 9 etapa estructura de costes la cual define los costes fijos y variables de nuestros procesos

\section{- Industria 4.0 y Transformación Digital:}

Actualmente estas 2 temáticas son la tendencia a nivel global por los cambios acelerados de la innovación de los procesos mas que en los productos, conociendo las 4 fases de la revolución industrial que son la 1 fase MECÁNICA nos dejó la producción en masa, 2 fase la electricidad cambiando nuestro consumo en la mayoría de los sectores, 3 fase AUTOMATIZACIÓN que obtuvo como base la tecnología de la información y ahora surge una 4 fase INTELIGENCIA ARTIFICIAL ROBOTICA BIG DATA esta etapa hace un paso más allá de la tecnología básica que estábamos acostumbrados a ver, el ejemplo más básico es la manufactura adictiva que con la tecnología construye objetos tridimensionales, estas impresoras pueden construir desde objetos funcionales como hasta comida comestible y la trasformación digital es ese movimiento de la captura de la información, los medios que podemos llegar a nivel global, mejorar la capacidad de uso y la eficiencia de los recursos. Es tanta la importación de esta herramienta que en nuestros productos podemos recopilar información de nuestro cliente a tal punto que sabremos con qué frecuencia lo utiliza, cada cuanto hace compra del producto entre otros, la importancia de la información es muy alta y no solo es valioso para nosotros, para todos los productos complementarios o skateholders. La problemática que se puede evidenciar en estos cambio es que muchas de las empresas tiene un trabajos operativo en las organizaciones, lo que va generar un desplazamiento del recurso humano afectándonos una vez más en la revolución industrial de muestro pasado, las empresas deben centrarse en que estas transformaciones les dará fortalezas competitivas con el mercado e internamente siendo más efectivos en los procesos, ese recurso humano debe ser capacitado para que los trabajos operativos pasen a trabajos intelectuales, generar oportunidades para la expansión de las empresas y no ver como la reducción de la nómina una optimización de los recursos

\section{- $\quad$ Liderazgo y talentos en las empresas del siglo XXI.}

En esta sesión realizamos actividades de coaching, para identificar el liderazgo en diferentes situaciones que se desarrollan en las organizaciones, como puede influir la actitud de las personas en el desarrollo de las actividades y el compromiso de cada una con el rol que desarrolla en la organización.

Trabajamos en que esperábamos cada uno de la sesión, e identificamos factores para poder desarrollar el liderazgo en un grupo de trabajo, ya sea grande o pequeño. 
Nos explicaban que un líder debe en las oportunidades en el lugar de la persona para poder entender el comportamiento, debe saber escuchar y dar la oportunidad de desarrollarse en el lugar donde se sienta mejor.

Se desarrollo una actividad en grupos donde todos teníamos que ganar sea el equipo A o el B y por parte de un grupo se genero una competencia para poder ganar el cual no era el objetivo sino solo poner a trabajar a todos los participantes del grupo y desarrollar la actividad donde los dos equipos obtuvieran la misma cantidad de puntos, evidentemente gano el equipo que se dedico a competir hasta el final. Con esta actividad tuvimos varias enseñanzas tanto para las organizaciones como para el desarrollo individual de las personas:

1. Fue una actividad democrática, con observaciones y sugerencias claras para una elección, se logro escuchar a todas las personas para tomar la decisión.

2. Se trabajo en equipo ya que se conto con la aprobación de cada uno de los participantes para tomar la decisión.

3. Se retroalimento de las características de un líder para identificar quien cuenta con las características.

\section{- Innovación y Marketing en el Desarrollo de Negocios en el Mundo Digital:}

En la actualidad del mundo corporativo, la tecnología se ha convertido en parte de la cotidianidad del funcionamiento de las organizaciones ya que esta representa una herramienta que permite optimizar la mayor parte de los procesos tanto operativos como administrativos, y son las plataformas digitales los escenarios más concretos de mercado virtual en donde la innovación y el marketing se vuelven indispensables para lograr atraer a los clientes en este ámbito tan competitivo.

Según lo trabajado en el aula, el primer paso para generar mejoras a partir de las herramientas digitales en lo que se denominó "industria digital 4.0" consiste en generar a partir de la creación de recursos digitales procesos que hagan mas eficiente el funcionamiento de la empresa bajo los principios de anticipación, integración, precisión, simplificación, agilidad y autonomía dividiéndose en dos categorías como son las básicas que consisten en IA, Blockchain y el denominado internet de las cosas y por otro lado la categoría de aplicaciones que consiste en la robótica, la fabricación digital y la realidad virtual.

Lo anterior debe ser evaluado por la directiva de la organización ya que es esta quien debe planificar la creación de estas herramientas con el fin que se considere necesario ya que estas pueden beneficiar a la empresa en distintos aspectos tales como la innovación, la disminución de costes, mejoras en el proceso de selección de colaboradores, creación de nuevos sistemas de información, mejora en las políticas de seguridad, disminución de riesgos, menor impacto ambiental entre otros. 


\section{Anexos:}

Enviados al correo de revisión de los profesores Jairo y Luis, entrevistas realizadas a la profesora BELEN de la Universidad de Salamanca y al Profesor Leonardo Urrego de la Universidad Santo Tomas, sobre sus conocimientos acerca del liderazgo. 